\title{
Ayudas ergogénicas en jugadores de fútbol profesional: una Revisión Sistemática
}

\author{
Alejandro Almendáriz-Villarán', Antonio Jesús Sánchez-Oliver²
}

\author{
Universidad Pablo Olavide, 2Universidad de Sevilla \\ Email: aalmvil@alu.upo.es, asanchez38@us.es
}

RESUMEN: Existen muchos artículos que recomiendan el uso de suplementos nutricionales para jugadores de fútbol de nivel profesional, aunque se han realizado con una muestra diferente. El objetivo de esta revisión fue analizar qué estudios existen sobre los efectos de la suplementación deportiva en jugadores/as de fútbol profesional. Se realizó una búsqueda avanzada en la base de datos de Web of Science. Los descriptores según el MeSH fueron: footbal, soccer, sports nutricional sciences y dietary supplement y se añadieron los descriptores de ergogenic y supplement, con la siguiente ecuación de búsqueda: $T S=$ (football OR soccer) AND TS= (dietary supplement OR sports nutritional sciences $O R$ supplement $O R$ ergogenic). Fueron encontrados 338 artículos iniciales, de los cuáles fueron excluidos 133 por el año de publicación, 14 por el idioma y 36 por no ser artículos originales de intervención. 95 artículos fueron desechados por tratar sobre otra temática. Dejando 60 artículos, donde se eliminaron 18 artículos por no tratar sobre fútbol y 29 por no ser sobre jugadores de fútbol sénior profesionales. Quedaron 13 artículos para una cuarta criba donde fueron eliminados 5 estudios debido a que la categoría era considerada semi-profesional, con un resultado final de 8 artículos. La suplementación de astaxantina, ácido docosahexaenoico, alopurinol y monohidrato de creatina tienen efectos positivos que influyen en el rendimiento, aunque es necesario realizar futuras investigaciones que profundicen sobre esta temática.

PALABRAS CLAVE: ayudas ergogénicas, fútbol, jugadores profesionales, nutrición deportiva.

\section{Ergogenics aids in professional soccer: a Sistematic Review}

ABSTRACT: There are many articles that recommend the use of nutritional supplements for professional level football players, although they have been done with a different sample. The objective of this review was to analyze what studies exist on the effects of sports supplementation on professional football players. An advanced search was performed in the Web of Science database. The descriptors according to the MeSH were: footbal, soccer, sports nutritional sciences and dietary supplement and the descriptors of ergogenic and supplement were added with the following equation of search: TS = (football OR soccer) AND TS = Sciences OR supplement OR ergogenic). 338 initial articles were found, of which 133 were excluded for the year of publication, 14 for the language and 36 for not being original articles of intervention. 95 articles were discarded for dealing with another topic. Leaving 60 articles where 18 articles were deleted for not dealing with football and 29 for not being about senior professional soccer players. There were 13 articles for a fourth sieve where they 
were eliminated 5 studies because the category was considered semiprofesional with a final result of 8 articles. The supplementation of astaxanthin, docosahexaenoic acid, allopurinol and creatine monohydrate have positive effects that influence the performance, although it is necessary to carry out future research that delves into this topic.

KEY WORDS: Ergogenic aids, football, professional players, sports nutrition. 


\section{INTRODUCCIÓN}

El Comité Olímpico Internacional (COI) en su posicionamiento de 2018 define a un suplemento como un alimento, componente alimenticio, nutriente o compuesto no alimenticio que se ingiere intencionalmente además de la dieta de consumo habitual con el objetivo de lograr un beneficio específico de salud y / o rendimiento (Maughan, et al., 2018). El uso de suplementos dietéticos está muy extendido entre deportistas de diferentes deportes y niveles (Sánchez-Oliver y Grimaldi-Puyana, 2017; Ventura Comes, Sánchez-Oliver, Martínez-Sanz, y Domínguez, 2018), llegando incluso a la población general que practica actividad física de forma recreativa (Sánchez-Oliver, 2013; Sánchez-Oliver, Miranda-León, y Guerra-Hernández, 2008), sobre todo en los países desarrollados (European Communities, 2008). Existen situaciones determinadas en las que los suplementos dietéticos pueden resultar beneficiosos, siempre y cuando exista de base una alimentación saludable, y sigan las tres premisas fundamentales de la suplementación: legalidad, eficacia y seguridad (Mata, Sánchez-Oliver, Domínguez, y Villegas, 2018). Hay que tener en cuenta, además, que la utilización de los suplementos nutricionales variará según la situación específica del deportista y las características concretas de cada deporte o modalidad (Mata, Sánchez-Oliver, Domínguez, y Villegas, 2018).

Prestar atención a los aspectos nutricionales es una cuestión de vital importancia para aportar a cada deportista la energía necesaria para afrontar las demandas físicas de cada modalidad deportiva (Mohr, Krustrup, y Bangsbo, 2005). La nutrición y los suplementos nutricionales pueden ser útiles en la mejora del rendimiento deportivo en el fútbol (Cotán, Fernández, Mata, y Sánchez-Oliver, 2017).

Parece evidente la importancia de la suplementación deportiva en el fútbol, ya que existen múltiples estudios sobre los efectos de los suplementos deportivos o ayudas ergogénicas en el fútbol. Así, de este modo, Arent, Pellegrino, Williams, Difabio y Greenwood (2010) observaron algunas mejoras en el rendimiento físico y mejoras con la suplementación a corto plazo en el estrés oxidativo y en la reducción de la respuesta de creatina quinasa. Por el contrario, Jastrzebska, Kaczmarczyk y Jastrzebski (2016) estudiaron los efectos de la suplementación de la vitamina $D$ sobre el rendimiento para un entrenamiento interválico, observando que una suplementación de vitamina $D$ durante 8 semanas no parece tener una mejora en el entrenamiento de alta intensidad.

En relación a esto, una revisión sistemática sobre la suplementación de creatina en el fútbol concluyó que son necesarios más estudios para comprobar los efectos ergogénicos de ésta en la capacidad aeróbica y en la repetición de acciones de alta intensidad en el fútbol competitivo (Miny, Burrowes, y Jidovtseff, 2016). Por otra parte, Deminice, Rosa, Franco, Jordao y de Freitas (2013), observaron que la suplementación de creatina, en jugadores de fútbol inhibe el aumento de marcadores inflamatorios tras pruebas de sprint repetidas, pero no de marcadores relacionados con el estrés oxidativo.

Respecto a la suplementación con cafeína los resultados son dispares, por un lado, Shirali, et al (2016) hallaron que acompañada de carnitina puede tener efectos positivos en la pérdida del porcentaje de grasa, del peso corporal y prevenir los incrementos en los niveles de ácidos grasos libres, aunque aumentó los niveles de glucosa en ayunas. Del Coso et al. (2012) encontraron también efectos beneficiosos en la capacidad de sprint y en la distancia recorrida a alta intensidad. Por el contrario, Pettersen et al. (2014) no encontraron beneficios de la suplementación de cafeína en el rendimiento.

Cockburn, Bell, y Stevenson (2013), en un estudio con jugadores semiprofesionales hallaron que la suplementación con carbohidratos y proteínas de leche 
después del ejercicio limita la disminución del rendimiento de sprint, de la agilidad y de la capacidad de realizar sprints repetidos durante la simulación fisiológica de los deportes de equipo de campo.

Laurenti et al. (2015), realizaron un meta-análisis sobre el uso de suplementos y la mejora del rendimiento en atletas donde se consideró que era importante profundizar sobre los efectos reales de los suplementos y los riesgos de su ingesta. Otra revisión de Russell y Kingsley (2014), sobre la eficacia de las intervenciones nutricionales en las habilidades de fútbol, estudió la ingesta de carbohidratos principalmente, además de la ingesta de cafeína y de líquido para mantener las habilidades en situaciones de fatiga, concluyendo que la eficacia dependerá de diversos factores como la dosis, el modo de administración, la respuesta individual de los sujetos y los cambios fisiológicos concretos de un ejercicio de fútbol.

Según Dickinson y Makay (2014), los conocimientos que tienen los consumidores de suplementos sobre la eficacia, la seguridad y las necesidades reales no están claros. De hecho, en los atletas el uso de suplementos para la mejora del rendimiento es generalizado, pese a que no existe una evidencia completa sobre su seguridad, eficacia y los mecanismos de acción, por lo que la necesidad de recopilar evidencia científica para aclarar estos aspectos está creciendo.

Las recomendaciones sobre el uso de suplementos deportivos no están claras, algunos artículos recomiendan el uso de suplementos para jugadores de nivel profesional, aunque la mayoría se han realizado con una muestra de nivel amateur 0 semi-profesional. Esto puede llevar a una recomendación errónea sobre el uso de suplementos deportivos en los jugadores de fútbol de nivel profesional, ya que la población diana no se corresponde con la muestra de los estudios. En base a la literatura revisada, existe poca evidencia científica respecto a la suplementación deportiva en jugadores de fútbol profesionales, por ello, el objetivo principal de esta revisión fue analizar que estudios existen sobre los efectos de la suplementación deportiva y las ayudas ergogénicas en jugadores/as de fútbol sénior de nivel profesional.

\section{MÉTODOS}

Estudio descriptivo de revisión sistemática tipo PRISMA (Preferred Reporting Items for Systematic reviews and Meta-Analyses) (Urrutia y Bonfill, 2010), sobre la influencia de las ayudas ergogénicas sobre el rendimiento en jugadores/as de fútbol sénior profesionales.

\subsection{Criterios de elegibilidad}

Se aplicaron los siguientes criterios de inclusión de los artículos encontrados, excluyendo los que no cumplieran los mismos: artículos originales publicados entre 2010 y septiembre de 2017, artículos escritos en inglés, ensayos clínicos aleatorizados, jugadores/as profesionales de fútbol de categoría sénior e influencia de suplementos 0 ayudas ergogénicas y nutricionales en el rendimiento de forma directa o indirecta. Siguiendo los criterios de inclusión se analizaron un total de 8 artículos.

\subsection{Fuentes de información y búsqueda}

Se realizó una búsqueda avanzada en la base de datos de Web of Science. Los descriptores utilizados se establecieron en base al MeSH: footbal, soccer, sports nutricional sciences y dietary supplement. Por otra parte, se añadieron los descriptores de ergogenic y supplement para completar la búsqueda, introduciendo todos los 
descriptores como (tema=TS) en la búsqueda avanzada de Web Of Science, de forma que se aplicó la siguiente ecuación de búsqueda:

$T S=$ (football OR soccer) AND TS= (dietary supplement OR sports nutritional sciences OR supplement OR ergogenic).

\section{RESULTADOS}

\subsection{Selección de artículos}

Un total de 338 resultados respondieron a la estrategia de búsqueda empleada, de los cuáles fueron excluidos 133 por el año de publicación del artículo, 14 por no ser en inglés y 36 por no ser artículos originales de intervención. Tras analizar el título y el resumen de los artículos, 95 fueron desechados por tratar sobre otra temática. De los 60 artículos restantes y, tras leer la metodología del estudio se eliminaron 18 artículos por descubrir que no eran sobre la especialidad deportiva de fútbol y 29 por no ser sobre jugadores de fútbol profesionales de categoría sénior. Los 13 artículos resultantes se analizaron en profundidad para ver que cumplían con los criterios de exclusión e inclusión, eliminando 5 artículos debido a que la categoría en la que jugaban los sujetos era considerada semi-profesional. De esta forma, tras un análisis exhaustivo de los artículos, se seleccionaron un total de 8 artículos como base de esta revisión (Figura 1).

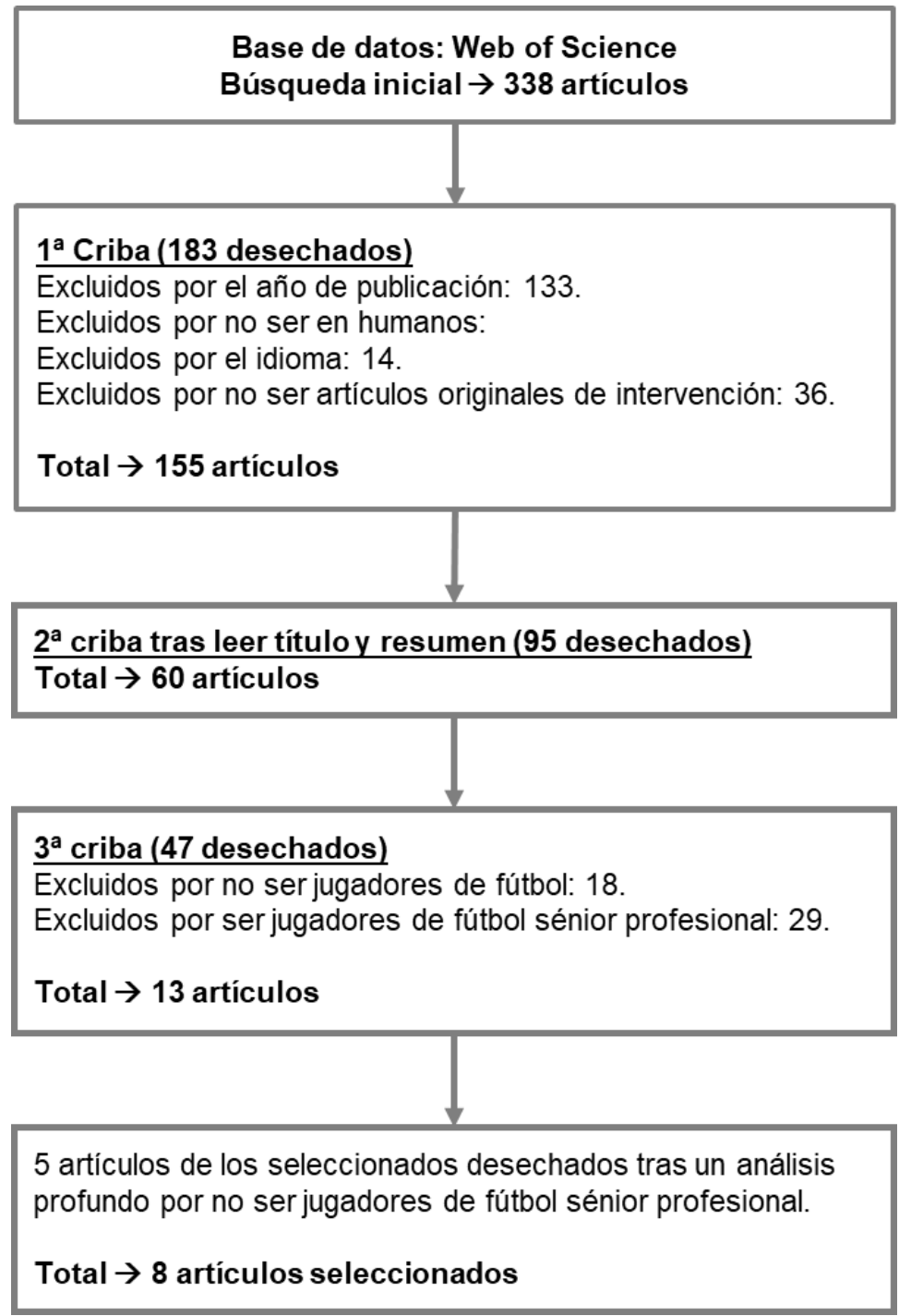


Figura 1. Fase de selección de los artículos científicos.

\subsection{Características de los estudios}

Los estudios hallados se han realizado con futbolistas profesionales de categoría sénior, estando toda la muestra compuesta por hombres, excepto en el estudio de Guzmán et al. (2011), donde participaron jugadoras de fútbol. Se consideró como nivel profesional a los clubes de primera y segunda división de sus respectivas ligas profesionales nacionales y los clubes que participaron en campeonatos profesionales.

Respecto al diseño, se encontraron 5 estudios aleatorizados doble ciego, (Baralic et al., 2015; Bassini et al., 2013; Claudino et al., 2014; Guzmán et al., 2011; Sanchis-Gomar, Salvagno y Lippi, 2014), 2 estudios aleatorizados en base a las posiciones de los jugadores con una administración de la suplementación de manera doble ciega, (Lollo, Amaya-Farfán, y De Carvalho-Silva, 2011; Lollo et al., 2014), y 1 estudio aleatorizado según la edad y el nivel de condición física, (Gunnarsson et al., 2013). En los artículos encontrados varios de ellos midieron los efectos directos sobre el rendimiento de la suplementación deportiva, (Claudino et al., 2014: Guzmán et al., 2011; Lollo et al., 2011; Lollo et al., 2014). Mientras que otros estudios midieron los efectos de la suplementación deportiva sobre parámetros que afectan indirectamente en el rendimiento deportivo (Baralic et al. 2015, Bassini et al. 2013, Gunnarsson et al. 2013; Sanchis-Gomar et al. 2014).

En cuanto a las ayudas nutricionales o suplementos utilizados, se utilizó: astaxantina, aceite rico en ácido docosahexaenoico, proteínas (proteína de suero, proteína de suero hidrolizada o caseína), cafeína, dieta enriquecida con proteína de suero y carbohidratos, proteína de suero hidrolizada, alopurinol y monohidrato de creatina (Baralic et al. 2015; Bassini et al. 2013; Claudino et al. 2014; Gunnarsson et al. 2013; Guzmán et al. 2011; Lollo et al. 2011; Lollo et al. 2014; Sanchis-Gomar et al. 2014).

\subsection{Síntesis de resultados}

La Tabla 1 recoge los autores, el año, la suplementación usada y la forma de administración de los suplementos, las pruebas y test realizados para valorar el posible efecto del suplemento y los resultados más relevantes de los 8 trabajos seleccionados en la revisión. Cabe destacar que la evidencia científica de los 8 artículos seleccionados en la revisión procede al menos de un ensayo clínico aleatorizado, por lo que se habla de un nivel de evidencia $A(\mathrm{lb})$. 
Autor/es

(año)

\begin{tabular}{|c|c|}
\hline $\begin{array}{l}\text { Baralic et al. } \\
(2015) .\end{array}$ & $\begin{array}{l}\text { Edad: } 17,9 \pm 0,2 \\
n=40 \text { hombres } \\
\text { jóvenes } \\
\text { Grupo: Asx } n=21 \text {, } \\
\text { Placebo } n=19 \\
\text { Club de Partizan de } \\
\text { Belgrado }\end{array}$ \\
\hline
\end{tabular}

$\mathrm{n}=34$ mujeres

Guzmán et Levante U.D. $n=20$,

al. (2011). Valencia C.F. $n=14$

Liga de fútbol

femenina española.

$\begin{array}{ll} & \\ \text { Lollo et al. } & \mathrm{n}=24 \text { hombres } \\ \text { (2011). } & \text { Primera división de } \\ & \text { Brasil. }\end{array}$

Bassini et al $\mathrm{n}=19$ hombres

(2013). La mayor liga de la

confederación

brasileña de fútbol

Tabla 1. Características de los estudios seleccionados.

\section{Suplementación}

Ingesta de una cápsula de $4 \mathrm{mg}$ de Astaxantina (Asx) durante 90 días.

Entrenamiento de 5-7 sesiones por semana (10-15 h semanales) y participación en el campeonato nacional.

Ingesta de la suplementación 3.5 $\mathrm{g} /$ dia de aceite de pescado rico en ácido docosahexaenoico

(DHA) $(n=12)$ o aceite de oliva $(\mathrm{n}=12)$ mediante 5 cápsulas diarias en el desayuno durante 4 semanas de entrenamiento. Ingesta de $1 \mathrm{~g} / \mathrm{kg} /$ día de proteína de suero (WP), proteína de suero hidrolizada (HWP) o caseína (CAS) inmediatamente después de sesión de entrenamiento durante 8 semanas. 1-2 partidos por semana y 6-8 entrenamientos de 2,5 horas aproximadamente.

Ingesta de $5 \mathrm{mg} / \mathrm{kg}$ de cafeína o lactosa (control) durante 1 sesión, se les proporcionó un desayuno específico suplementado con cafeína o lactosa.

El grupo con cafeína se dividió en 2 grupos (CEx y SCEx) dependiendo de los niveles séricos de cafeína (<900\% y $>10.000 \%$, respectivamente)

\section{Pruebas y test}

Muestra de sangre y saliva antes de entrenamiento matinal en ayunas al inicio del estudio y tras los 90 días de suplementación.

2 semanas previas a la primera sesión se obtuvieron valores de VO2 máx. composición corporal y datos demográficos.

Mediciones (pre y post-tratamiento) de tiempo de reacción complejo y precisión. Presión en diferentes botones y pedales con las manos y los pies izquierdos y derechos, o dejar de responder, según estímulos visuales y auditivos.

Medición de características antropométricas, rendimiento físico (yoyo test y prueba de $3000 \mathrm{~m}$ ), y varias variables bioquímicas en sangre (ácido úrico, colesterol total, colesterol HDL, creatinina, glucosa), antes y después del período experimental.

Realización de 45 minutos de ejercicio intermitente seguido de un test intermitente de recuperación (yoyo test) hasta el agotamiento.

\section{Resultados}

Aumento en saliva de inmunoglobulina $A$ secretora $(\lg A)$ en reposo junto una disminución en el equilibrio prooxidante-antioxidante.

Reducción significativa de los niveles de enzimas de los músculos plasmáticos mediante suplementos de Asx y un entrenamiento regular. Aumento en el recuento de neutrófilos y el nivel de hs-PCR sólo en el grupo placebo.

Diferencias no significativas en la ingesta alimentaria de ambos equipos.

En el grupo DHA, el tiempo de reacción complejo y la precisión disminuyeron de forma similar, y en consecuencia aumentó la eficiencia de la reacción compleja.

Aumento de masa muscular con la caseína ( $p$ $<0,039)$, WP y HWP favorecieron el mantenimiento de la masa muscular inicial. No mejoró el rendimiento físico respecto a los tratamientos aplicados. No causó anomalías en las variables bioquímicas y antropométricas monitoreadas, aunque si en los cambios antropométricos adversos.

La suplementación con cafeína no afectó significativamente el rendimiento.

El ejercicio cambió las concentraciones sanguíneas de varios aminoácidos y aumentó las concentraciones séricas de amoníaco, glucosa, lactato e insulina.

Aumento inducido por el ejercicio de valina (similar al 29\%), que fue inhibido por la cafeína. En respuesta al ejercicio, no aumentó la uremia y mostraron un aumento significativamente menor en sus concentraciones séricas de arginina $(15 \%)$, citrulina (16\%) y ornitina (ND). 


\begin{tabular}{|c|c|c|c|c|}
\hline $\begin{array}{l}\text { Gunnarsson } \\
\text { et al. (2013). }\end{array}$ & $\begin{array}{l}\text { Edad media } 24 \pm 1 \\
\mathrm{n}=19 \text { hombres } \\
1^{\text {a }} \text { y } 2^{\mathrm{a}} \text { división de la } \\
\text { liga danesa. }\end{array}$ & $\begin{array}{l}\text { Ingesta de dieta con alto } \\
\text { contenido de carbohidratos y } \\
\text { proteína de suero }(n=9) \text { y dieta } \\
\text { normal }(n=7) \text {. } \\
\text { Dieta durante un período de } \\
\text { recuperación de } 48 \text { horas } \\
\text { después de } 2 \text { partidos de } 60 \text { min } \\
\text { y } 90 \text { min en días separados. } \\
\text { G.control (CON), G.con hidratos } \\
\text { y proteína de suero (HCP), } \\
\text { G.partido simulado de } 90 \text { min } \\
\text { (SIM90) y de } 60 \text { min (SIM60) }\end{array}$ & $\begin{array}{l}15 \text { minutos después, así como } 24 \text { y } 48 \mathrm{~h} \\
\text { después de cada partido, muestra de } \\
\text { sangre y una biopsia del vasto lateral. } \\
\text { Medición en el laboratorio más de } 48 \mathrm{~h} \\
\text { después de la última sesión y al menos } 2 \\
\text { h después de la ingestión de una comida. } \\
\text { Se midió la absorción máxima de oxígeno } \\
\text { (VO2max) alcanzado durante } 30 \\
\text { segundos, y la frecuencia cardiaca } \\
\text { máxima en una prueba incremental } \\
\text { exhaustiva en rodillo. }\end{array}$ & $\begin{array}{l}\text { Disminución del glucógeno muscular }(\mathrm{P}<0,05) \\
\text { en } 54,48,53 \text { y } 38 \% \text { después de los partidos en } \\
\text { CON, HCP, SIM90 y SIM } 60 \text {, respectivamente. } \\
\text { La resíntesis de glucógeno durante las primeras } \\
48 \text { h después del ejercicio no fue diferente entre } \\
\text { CON y HCP, mientras que la resíntesis de } \\
\text { glucógeno fue más lenta }(P<0,05) \text { durante las } \\
\text { primeras } 24 \text { h después de SIM60 que SIM90 } \\
\text { ( } 2,88+/-0,84 \text { vs } 4,32+/-0,54 \text { mmol / kgdw } \\
\text { /marido). En HCP, el contenido de glucógeno en } \\
\text { las fibras musculares de tipo II se redujo todavía } \\
48 \text { horas después del partido. }\end{array}$ \\
\hline $\begin{array}{l}\text { Lollo et al. } \\
\text { (2014). }\end{array}$ & $\begin{array}{l}\text { Edad: } 18 \pm 0,8 \\
\mathrm{n}=24 \text { hombres } \\
\text { jóvenes } \\
\text { Torneo oficial de la } \\
\text { mejor liga brasileña }\end{array}$ & $\begin{array}{l}\text { Ingesta } 0,5 \mathrm{~g} \text { de proteína/kg/día, } \\
\text { a doble ciega con proteína de } \\
\text { suero (WP), proteína de suero } \\
\text { hidrolizada (HWP), o un placebo } \\
\text { no proteico (maltodextrina - } \\
\text { MALTO), inmediatamente antes } \\
\text { y después del entrenamiento en } \\
12 \text { semanas y en los días de } \\
\text { descanso. Dosis de proteína de } \\
\text { la dieta y suplemento = } 15 \% \text { de } \\
\text { la ingesta calórica diaria total. }\end{array}$ & $\begin{array}{l}\text { Evaluaciones realizadas el mismo día con } \\
\text { medidas antropométricas y de rendimiento } \\
\text { ( } 2 \text { días antes de la intervención y } 2 \text { días } \\
\text { después). } \\
\text { Realización de test Yo-Yo 2, prueba de } \\
\text { esfuerzo máximo de } 4 \text { minutos, salto en } \\
\text { cuclillas, contramovimiento y } \\
\text { contramovimiento con manos }\end{array}$ & $\begin{array}{l}\text { Disminución significativa con la ingesta de HWP } \\
\text { en los indicadores de daño muscular, creatina } \\
\text { quinasa (-42\%) y lactato deshidrogenasa (-30\%). } \\
\text { La suplementación WP no mostró cambios } \\
\text { significativos en estos indicadores en } \\
\text { comparación con el grupo MALTO. } \\
\text { La masa muscular no mostró cambios, y el } \\
\text { rendimiento físico en una prueba aeróbica } \\
\text { disminuyó en el grupo de HWP. }\end{array}$ \\
\hline $\begin{array}{l}\text { Sanchis- } \\
\text { Gomar et al. } \\
\text { (2014). }\end{array}$ & $\begin{array}{l}\text { Edad: } 25 \pm 2 \\
\mathrm{n}=12 \text { hombres } \\
\text { La } 1^{\text {a }} \text { división de la } \\
\text { liga española. }\end{array}$ & $\begin{array}{l}\text { Ingesta de } 300 \text { mg de alopurinol } \\
(n=6) 4 \text { horas antes de un partido } \\
\text { de la Liga Española de Fútbol, } \\
\text { grupo control recibió placebo } \\
(n=6) \text { (celulosa). }\end{array}$ & $\begin{array}{l}\text { Extracción de muestras de sangre antes } \\
\text { del partido y doce horas después. } \\
\text { después de un ayuno durante la noche, a } \\
\text { la misma hora y condiciones (temperatura } \\
\text { y humedad), después de que los sujetos } \\
\text { permanecieran sentados durante } 10 \mathrm{~min} \text {. }\end{array}$ & $\begin{array}{l}\text { Disminución significativa con la ingesta de } \\
\text { alopurinol en las concentraciones séricas de } \\
\text { ácido úrico después del partido. } \\
\text { Aumento significativo en suero de: calcio y } \\
\text { concentraciones de25(OH)D-3 en grupo } \\
\text { suplementado. }\end{array}$ \\
\hline $\begin{array}{l}\text { Claudino et } \\
\text { al. (2014). }\end{array}$ & $\begin{array}{l}\text { Edad: } 18,3 \pm 0,9 \\
\mathrm{n}=14 \text { hombres } \\
\text { Equipo Red Bull } \\
\text { Brasil } \\
1^{\text {a }} \text { división de la } \\
\text { Copa Partisan de } \\
\text { Brasil. }\end{array}$ & $\begin{array}{l}\text { Ingesta de } 20 \mathrm{~g} \text { al día durante } 1 \\
\text { semana y } 6 \text { semanas con } 5 \mathrm{~g} \\
\text { diarios de suplementación con } \\
\text { monohidrato de creatina o } \\
\text { placebo. } \\
\text { Durante la pretemporada ( } 7 \\
\text { semanas), todos los sujetos } \\
\text { fueron sometidos a un } \\
\text { entrenamiento de fútbol físico y } \\
\text { específico. }\end{array}$ & $\begin{array}{l}\text { Efectos en la fuerza muscular de las } \\
\text { extremidades inferiores. La potencia } \\
\text { muscular de las extremidades inferiores } \\
\text { se midió por el rendimiento de salto en } \\
\text { contramovimiento. } \\
\text { El rendimiento de salto se comparó entre } \\
\text { los grupos en la línea de base }(p=0,99) \text {. }\end{array}$ & $\begin{array}{l}\text { Aumento del rendimiento en el grupo de creatina, } \\
\text { pero no fue significativo ( } \mathrm{P}=0,23 \text { para la } \\
\text { interacción tiempo por grupo). } \\
\text { Mayor proporción de sujetos que experimentaron } \\
\text { una menor reducción en el rendimiento de saltos } \\
\text { de forma significativa en el grupo de creatina ( } 5 \text { y } \\
1 \text {, respectivamente, } p=0,05) \text { después del } \\
\text { entrenamiento. } \\
\text { Efecto negativo del placebo }(50 \%) \text { en el } \\
\text { rendimiento de salto y la suplementación con } \\
\text { creatina produjo un efecto trivial muy probable } \\
(96 \%) \text {. }\end{array}$ \\
\hline
\end{tabular}




\section{DISCUSIÓN}

Esta revisión pone de manifiesto la dificultad para encontrar evidencia dedicada a los efectos de la suplementación deportiva sobre el rendimiento en jugadores/as sénior de nivel profesional. Además de los pocos estudios encontrados, dentro de estos existe una gran diversidad en cuanto a los suplementos y la metodología usados, dando lugar a hallazgos muy dispares. Cada estudio tiene unas características y metodología concretas, siendo complicado encontrar coincidencias entre los estudios que permitan generalizar los resultados encontrados.

Baralic et al. (2015) hallaron que la suplementación con astaxantina atenúa los daños musculares evitando la inflamación inducida tras un entrenamiento y mejora los niveles de salivación IgA en reposo que está relacionada con una disminución en el equilibrio prooxidante-antioxidante. En cuanto a la validez metodológica del estudio se trata de un estudio aleatorizado doble ciego, aunque la heterogeneidad de los grupos podría influir en los resultados. Por otra parte, parece que la administración de la suplementación no fue individualizada y habría que analizar en si es necesario individualizar la dosis. La muestra estaba compuesta por 40 jugadores con una edad media de $17,9 \pm 0,2$, aunque el estudio los califica como jugadores profesionales, existe el riesgo de que puedan ser jugadores muy jóvenes, por lo que limita los resultados de este estudio.

Guzmán et al. (2011) analizaron los efectos de la suplementación de aceite de pescado rico en ácido docosahexaenoico en el tiempo de reacción complejo, la precisión y la eficiencia, observando que dicha suplementación puede mejorar los tiempos de reacción y de precisión y, por lo tanto, aumentando la eficiencia. Esta intervención se lleva a cabo en una muestra compuesta por 34 jugadoras, realizando un estudio aleatorizado doble ciego, donde habría que tener en cuenta que los resultados pueden variar en el caso de ser hombres al tener exigencias fisiológicas distintas. Al parecer no se encontraron diferencias nutricionales entre las jugadoras, pero se debería analizar si en estos resultados pueden influir en los diferentes entrenamientos sobre la prueba de agilidad, al ser de clubes distintos.

Lollo et al. (2011), en una intervención con 24 jugadores, investigaron los efectos sobre la composición corporal, los parámetros bioquímicos y el rendimiento de la ingesta tras el entrenamiento de 3 tipos de suplementos de proteínas diferentes (proteína de suero, proteína de suero hidrolizada y caseína). Se trata de un estudio aleatorizado en base a las posiciones de los jugadores, con una administración de los suplementos de manera doble ciega. En este estudio ningún tipo de proteína influyó en el rendimiento, aunque la ingesta de proteína de suero y de proteína de suero hidrolizada tuvo efectos positivos en el mantenimiento de la masa muscular y, la caseína en el aumento de la misma. En esta intervención la validez de la muestra estaría limitada, debido a la edad de los jugadores y a la falta de información referente al club al que pertenecían los sujetos, así como alguna otra variable contaminante no tenida en cuenta.

Estos datos no coinciden con el estudio de Lollo et al. (2014), donde analizaron los efectos de la ingesta antes y después del entrenamiento de proteína de suero hidrolizada sobre el rendimiento en comparación con la proteína de suero y la maltodextrina (placebo no proteico). En esta intervención el grupo experimental (proteína de suero hidrolizada) no mostró cambios en la composición corporal, pero si mejoró el rendimiento en una prueba aeróbica y en los marcadores de daño muscular, por lo que se propone que la ingesta de proteína de suero hidrolizada puede disminuir el daño muscular. En cuanto al diseño, el estudio es similar al de Lollo et al. (2011), con una aleatorización de grupos en base a las posiciones de los jugadores y con los 
suplementos administrados de forma doble ciega. Respecto a la validez de la muestra sucede los mismo que con la intervención de Lollo et al. (2011) y Baralic et al. (2015), se trata de una muestra muy joven. Además, al igual que el estudio de Lollo et al. (2011), existe falta de información sobre el club de procedencia, pudiendo afectar a la validez de la muestra.

Bassini et al. (2013) investigaron los efectos de la ingesta de cafeína. En este estudio el grupo suplementado no obtuvo mejoras en el rendimiento. Parece que la cafeína disminuye la concentración sérica de glutamina que a su vez disminuye el transporte de aminoácidos al hígado, conllevando una disminución de la síntesis de urea. El estudio fue aleatorizado doble ciego, por lo que a priori, la validez es adecuada, aunque no menciona al equipo y sus características, coincidiendo con Lollo et al. (2011) y Lollo et al. (2014), por lo que habría que tener esta cuestión en cuenta. Se podría plantear que para este tipo de intervención la suplementación de cafeína no muestra una mejora sobre el rendimiento.

Gunnarson et al. (2013) analizaron los efectos de una dieta enriquecida con carbohidratos y proteína de suero sobre la resíntesis de glucógeno. En este estudio no encontraron mejoras significativas, es decir, la resíntesis de glucógeno $48 \mathrm{~h}$ después de un partido de fútbol no se eleva por la ingestión de una dieta enriquecida con carbohidratos y proteína de suero respecto a una dieta no enriquecida. Aunque la muestra se dividió aleatoriamente en dos grupos en base a la edad y el nivel de condición física, el grupo experimental contaba con 9 sujetos y el grupo control con 7 , siendo una limitación importante para la validez del estudio.

Sanchis-Gomar et al. (2014) estudiaron los efectos de la suplementación de alopurinol. Esto puede tener efecto en algunos parámetros que pueden afectar al rendimiento, de hecho, se concluyó que la suplementación de vitamina $\mathrm{D}$ puede ser un fármaco eficaz para disminuir la hipovitaminosis $D$ y la hiperuricemia, debido a que el grupo experimental mostró tras el partido una disminución de las concentraciones séricas de ácido úrico y un aumento de los niveles de calcio y de 25(OH)D3. En cuanto al diseño, se trata de un estudio aleatorizado doble ciego controlado, por lo que la validez metodológica y el nivel de los jugadores parecen adecuados, aunque se debería considerar que la dosis de suplementación de alopurinol no fue de forma individualizada.

En otro estudio, Claudino et al. (2014) investigaron los efectos de la suplementación de monohidrato de creatina. Los datos sugieren que dicha suplementación puede disminuir los decrementos de fuerza tras un entrenamiento progresivo de pre-temporada. Sería necesario analizar si tendría los mismos efectos en un entrenamiento durante la competición y, si tendrían los mismos efectos con una suplementación individualizada o con distinta carga. La validez de diseño parece adecuada, ya que se trata de un estudio aleatorio doble ciego controlado con placebo de grupos paralelos. Como factor limitante y aunque el artículo los menciona como jugadores de élite, habría que analizar en profundidad si estos datos se pueden extrapolar a jugadores de nivel profesional, ya que no queda claro en el texto.

Como se han mencionado anteriormente, de los estudios escogidos en la revisión, se excluyeron 5 publicaciones por contener una muestra de nivel semiprofesional, de las cuales 2 se excluyeron porque la muestra pertenecía a categoría juvenil de fútbol profesional (Pettersen et al. 2014 y Jordan et al. 2014), 2 porque la categoría era considerada semi-profesional (Cockburn et al. 2013; Deminice et al. 2013 y 1 porque la muestra total no era profesional y el diseño fue cuasi-experimental (Villanueva et al. 2011). 
En el estudio de Villanueva et al. (2011), se incluyeron jugadores profesionales de la primera división de la liga española de fútbol, pero estos pertenecían sólo al grupo experimental, comparándose con un grupo control compuesto de jugadores de tercera división de la liga española, siendo un factor que limita mucho la validez del estudio y, no cumpliendo los criterios para ser incluidos en esta revisión. En el estudio midieron los efectos de la suplementación de $80 \mathrm{mg}$ de hierro sobre el metabolismo hematológico y del hierro durante 3 semanas de pre-temporada. Parece que la suplementación de hierro aumentó los valores de hierro sérico, ferritina sérica y saturación de transferrina, así como mayores valores de hematocrito, volumen corpuscular medio, ferritina sérica y saturación de transferrina, pudiendo influir indirectamente en el rendimiento físico. A pesar de que se recomienda la suplementación de hierro para jugadores de élite, los resultados no están claros dado que se trata de un diseño cuasi-experimental. Por otra parte, se midieron los parámetros (pre y post tratamiento) sólo con el grupo experimental, mientras que con el grupo control se midió sólo después del tratamiento. Además, el número de sujetos asignados a cada grupo no era homogéneo, $n=24$ en el grupo experimental y $n=11$ en el grupo control. Todos estos parámetros ponen en duda la fiabilidad del estudio, por lo que se recomienda profundizar sobre esta temática con un diseño experimental y con una muestra que sea exclusivamente profesional.

En vista a lo hallado con un meta-análisis de Knapik et al. (2016), sobre la prevalencia del consumo de suplementos dietéticos en atletas, resulta complicado generalizar los datos del estudio en deportistas de élite debido a la falta de homogeneidad entre los diferentes estudios, considerando que son necesarios futuros estudios que mejoren la metodología. Del mismo modo, el presente estudio coincide con este meta-análisis en la dificultad para encontrar estudios realizados con jugadores/as de fútbol profesionales, en el que sólo se encontraron en la modalidad de fútbol 4 estudios de jugadores de fútbol de élite.

Por último, al comparar la validación de los suplementos utilizados en los estudios en base al Instituto Australiano del Deporte (AIS, 2017), se puede observar que pertenecen a los suplementos validados (grupo A), los suplementos de monohidrato de creatina, de proteínas (proteína de suero, proteína de suero hidrolizada y de caseína), cafeína y dieta enriquecida con carbohidratos y proteínas. El ácido docosahexaenoico estaría dentro del grupo $\mathrm{B}$ y el alopurinol y la astaxantina estarían dentro del grupo $\mathrm{C}$ de suplementación, dado que no se encuentran en el grupo A y B. Cabe mencionar que el AIS es un organismo donde el enfoque de la suplementación deportiva es a nivel genérico, no obstante, aunque no trate exclusivamente sobre el fútbol, nos puede aportar una idea sobre la calidad o validación de los suplementos utilizados en los estudios.

\subsection{Limitaciones del estudio}

Entre las principales limitaciones de los estudios seleccionados se encuentra la validez de la muestra en los estudios de Baralic et al. (2015), Lollo et al. (2011) y Lollo et al. (2014), donde ésta estaba compuesta por jóvenes jugadores profesionales de fútbol que, a excepción del estudio de Baralic et al. (2015), no especifican el club del que provienen, solamente la competición de forma general. Por otra parte, en cuanto a la validez en la composición de la muestra, los estudios de Baralic et al. (2015) y Gunnarsson et al. (2013) no contienen una muestra homogénea, con un número de participantes diferentes en cada grupo. Respecto al diseño, habría que mencionar el diseño del estudio de Gunnarson et al. (2013), dado que no se administró de manera doble ciega la dieta enriquecida con carbohidratos y proteínas que debían ingerir los jugadores. 
Igualmente, habría que tener en cuenta que la revisión sistemática sólo se ha utilizado una base de datos (Web Of Science) y que, por lo tanto, sería conveniente añadir otras bases de datos que pueden contener artículos relacionados con la temática, como Medline o SportDiscus. Por último, mencionar que ha sido necesario modificar algunos puntos de la metodología tipo PRISMA, pudiendo repercutir en la calidad de la presente revisión sistemática.

Existen pocos estudios sobre jugadores/as de fútbol profesional, aunque el problema es mayor en el caso de las jugadoras de fútbol, donde la falta de estudios es mucho más evidente, por lo que, habría que realizar futuras investigaciones que profundicen sobre el uso de suplementos deportivos en jugadores/as de fútbol sénior de nivel puramente profesional. Además, para futuras investigaciones se recomienda que se tengan en cuenta otros datos que pueden ser relevantes para la validez del estudio, como el nivel de los jugadores.

\section{CONCLUSIONES}

Respecto a los efectos de la suplementación sobre el rendimiento en jugadores de fútbol profesional, se han encontrado efectos positivos en el rendimiento la suplementación de astaxantina, ácido docosahexaenoico, alopurinol y monohidrato de creatina, por lo que, a falta de corroborar esto en posteriores y mejores estudios, habría indicios del efecto de éstos en jugadores profesionales de fútbol sobre las situaciones descritas (Baralic et al. 2015; Claudino et al. 2014; Guzmán et al. 2011; Sanchis-Gomar et al. 2014).

Por otra parte, la suplementación de proteína de suero y de proteína de suero hidrolizada tiene efectos positivos para mantener la masa muscular y la suplementación de caseína tiene efectos positivos para aumentar la masa muscular (Lollo et al., 2011). No obstante, respecto al rendimiento, los datos sobre la suplementación de proteína de suero hidrolizada no están claros, ya que en el estudio de Lollo et al. (2011) no encontró mejoras en el rendimiento y, Lollo et al. (2014) hallaron mejoras en el rendimiento de una prueba aeróbica y en los marcadores del daño muscular, siendo necesarios más estudios para profundizar sobre esta temática.

Los resultados de esta revisión ponen en manifiesto la dificultad para encontrar estudios que puedan extrapolar unos resultados claros para jugadores/as sénior de nivel profesional sobre los beneficios de la suplementación deportiva o las ayudas ergogénicas en el rendimiento deportivo. En cuanto al método, los suplementos encontrados en cada intervención y los protocolos de intervención son muy diferentes y en muchos casos los datos obtenidos no están del todo claros.

Por último, cabría recordar que algunos suplementos pueden ser beneficiosos para algunos deportistas en algunos contextos específicos, por lo que la suplementación debe ser individualizada y contextualizada. Los deportistas deben tomar decisiones informadas sobre el consumo de suplementos que maximicen los beneficios y minimicen los riesgos, contemplando el potencial de resultados positivos y negativos sobre su consumo.

\section{REFERENCIAS}

AIS (2017). Australian Sports Commission. Bruce, Australia: Australian Sports Commission. 
Arent, S. M., Pellegrino, J. K., Williams, C. A., Difabio, D. A., y Greenwood, J. C. (2010). Nutritional supplementation, performance, and oxidative stress in college soccer players. Journal of strength and conditioning research, 24(4), 1117-24.

Baralic, I., Andjelkovic, M., Djordjevic, B., Dikic, N., Radivojevic, N., Suzin-Zivkovic, V., Radojevic-Skodric, S., y Pejic, S. (2015). Effect of Astaxanthin Supplementation on Salivary IgA, Oxidative Stress, and Inflammation in Young Soccer Players. Evidence-based complementary and alternative medicine, 2015, 1-9.

Bassini, A., Magalhães-Neto, A. M., Sweet, E., Bottino, A., Veiga, C., Tozzi, M. B., ... \& Cameron, L. C. (2013). Caffeine Decreases Systemic Urea in Elite Soccer Players during Intermittent Exercise. Medicine and science in sports and exercise, 45(4), 683-90.

Claudino, J. G., Mezêncio, B., Amaral, S., Zanetti, V., Benatti, F., Roschel, H., Gualano, B., Amadio, A. C., y Serrão, J.C. (2014). Creatine monohydrate supplementation on lower-limb muscle power in Brazilian elite soccer players. Journal of the international society of sports nutrition, 11(32), 1-6.

Cockburn, E., Bell, P.G. y Stevenson, E. (2013). Effect of Milk on Team Sport Performance after Exercise-Induced Muscle Damage. Medicine and science in sports and exercise, 45(8), 1585-92.

Del Coso, J., Muñoz-Fernández, V.E., Muñoz, G., Fernández-Elías, V.E., Ortega, J.F., Hamouti, N., Barbero, J.C., Muñoz-Guerra, J. (2012). Effects of a CaffeineContaining Energy Drink on Simulated Soccer Performance. Plos One, 7(2), 1-8. DOI: $10.1371 /$ journal.pone.0031380.

Deminice, R., Rosa, F.T., Franco, G. S., Jordao, A. A., y de Freitas, E. C. (2013). Effects of creatine supplementation on oxidative stress and inflammatory markers after repeated-sprint exercise in humans. Nutrition, 29(9), 1127-32.

Dickinson A., y Makay D. (2014). Health habits and other characteristics of dietary supplement users: a review. Nutrition Journal, 13(1), 1-8.

European Communities (2008). Characteristics and perspectives of the market for food supplements containing substances other than vitamins and minerals. Bruselas, Bélgica: Comisión Europea. Recuperado de https://ec.europa.eu/food/safety/labelling_nutrition/supplements_en.

Gunnarsson, T.P., Bendiksen1, M., Bischoff, R., Christensen, P.M., Lesivig, B., Madsen, K., Stephens, F., Greenhaff, P., Krustrup, P. y Bangsbo, J. (2013). Effect of whey protein- and carbohydrate-enriched diet on glycogen resynthesis during the first $48 \mathrm{~h}$ after a soccer game. Scandinavian journal of medicine \& science in sports, 23(4), 508-15.

Guzmán, J.F., Esteve, H., Pablos, C., Pablos, A., Blasco, C. y Villegas, J.A. (2011). DHArich fish oil improves complex reaction time in female elite soccer players. Journal of sports science and medicine, 10(2), 301-5.

Jastrzebska, M., Kaczmarczyk, M. y Jastrzebski, Z. (2016). Effect of Vitamin D Supplementation on Training Adaptation in Well-Trained Soccer Players. Journal of strength and conditioning research, 30(9), 2648-55. 
Jordan, J.B., Korgaokar, A., Farley, R.S., Coons, J.M. y Caputo, J.L. (2014). Caffeine Supplementation and Reactive Agility in Elite Youth Soccer Players. Pediatric exercise science, 26(2), 168-76.

Knapik, J.J., Steelman, R.A., Hoedebecke, S.S., Austin, K.G., Farina, E.K. y Lieberman, H.R. (2016). Prevalence of Dietary Supplement Use by Athletes: Systematic Review and Meta-Analysis. Sports Med, 46(1), 103-23.

Laurenti, P., de Waure, C., De Meo, C., Terracciano, E., Di Nardo, F., Ricciardi, W. (2015). Does the use of dietary supplements enhance athletes' sport performances? A systematic review and a meta-analysis. Epidemiology Biostatistics and Public Health, 12(4), e11593:1-15.

Lollo, P.C., Amaya-Farfán, J. y De Carvalho-Silva, L.B. (2011). Physiological and Physical Effects of Different Milk Protein Supplements in Elite Soccer Players. Journal of human kinetics, 30, 49-57.

Lollo, P.C., Amaya-Farfan, J., Faria, I.C., Salgado, J.V.V., Chacon-Mikahil, M.P.T., Cruz, A.G., Oliveira, C.A.F., Montagner, P.C., Arruda, M. (2014). Hydrolysed whey protein reduces muscle damage markers in Brazilian elite soccer players compared with whey protein and maltodextrin. A twelve-week in-championship intervention. International dairy journal, 34(1), 19-24.

Mata, F., Sánchez-Oliver, A. J., Domínguez, R., \& Villegas, J. A. (2018). Suplementación en el deporte: directrices desde la responsabilidad profesional. Habilidad motriz: Revista de ciencias de la actividad fisica y del deporte, (51), 4-12.

Maughan, R. J., Burke, L. M., Dvorak, J., Larson-Meyer, D. E., Peeling, P., Phillips, S. M., ... \& Meeusen, R. (2018a). IOC consensus statement: dietary supplements and the high-performance athlete. International Journal of Sport Nutrition and Exercise Metabolism, 28(2), 104-125.

Minya, K., Burrowesb, J. y Jidovtseff, B. (2016). Interest of creatine supplementation in soccer. Science \& Sports 32(2), 61-72.

Mohr, M., Krustrup, P. y Bangsbo, J. (2005). Fatigue in soccer: A brief review. Journal of Sports Sciences, 23(6), 593-99.

Pettersen, S.A., Krustrup, P., Bendiksen, M., Randers, M.B., Brito, J., Bangsbo, J., Jin, Y. y Mohr, M. (2014). Caffeine supplementation does not affect match activities and fatigue resistance during match play in young football players. Journal of Sports Sciences, 32(20), 1958-65.

Russell, M. y Kingsley, M. (2014). The Efficacy of Acute Nutritional Interventions on Soccer Skill Performance. Sports Medicine, 44(7), 957-70.

Sánchez-Oliver, A. J. (2013). Suplementación nutricional en la actividad físico-deportiva: análisis de la calidad del suplemento proteico consumido. Granada: Universidad de Granada.

Sánchez-Oliver, A. J., y Grimaldi-Puyana, M. (2017). Análisis del consumo de suplementos nutricionales en jugadores de la liga EBA. Cuadernos de Psicología del Deporte, 17(3), 163-68. 
Sánchez-Oliver, A. J., Miranda-León, M. T., y Guerra-Hernández, E. (2008). Estudio estadístico del consumo de suplementos nutricionales y dietéticos en gimnasios. Archivos Latinoamericanos de Nutrición, 58(3), 221-7.

Sanchis-Gomar, F., Salvagno, G.L. y Lippi, G. (2014). Inhibition of Xanthine Oxidase and Exercise on Serum Uric Acid, 25(OH)D-3, and Calcium Concentrations. Clinical laboratory, 60(8), 1409-11.

Shirali, S., Daneghian, S., Hosseini, S.A., Ashtary-Larky, D., Daneghian, M. y Mirlohi, M.S. (2016). Effect of Caffeine Co-Ingested with Carnitine on Weight, Body-Fat Percent, Serum Leptin and Lipid Profile Changes in Male Teen Soccer Players: a Randomized Clinical Trial. International journal of pediatrics-mashhad, 4(10), 3685-98.

Urrutia, G. y Bonfill, X. (2010). Declaración PRISMA: una propuesta para mejorar la publicación de revisiones sistemáticas y metaanálisis. Med Clin, 135(11), 507-11.

Ventura Comes, A., Sánchez-Oliver, A., Martínez-Sanz, J., \& Domínguez, R. (2018). Analysis of Nutritional Supplements Consumption by Squash Players. Nutrients, 10(10), 1341.

Villanueva, J., Soria, M., González-Haro, C., Ezquerra, L., Nieto, J.L. y Escanero, J.F. (2011). Oral Iron Treatment Has a Positive Effect on Iron Metabolism in Elite Soccer Players. Biological trace element research, 142(3), 398-406. 